\title{
Locating a bioenergy facility using a hybrid optimization method
}

\author{
Athanasios A. Rentizelas*, Ilias P. Tatsiopoulos \\ Department of Mechanical Engineering, Sector of Industrial Management \& Operational Research, National Technical University of Athens, \\ 9 Iroon Polytechniou Str., Zografou, 15780 Athens, Greece
}

\section{A R T I C L E I N F O}

\section{Article history:}

Received 15 July 2008

Accepted 8 August 2009

Available online 21 August 2009

Keywords:

Facility location

OR in energy

Hybrid optimization

Bioenergy facility

Supply chain

\begin{abstract}
A B S T R A C T
In this paper, the optimum location of a bioenergy generation facility for district energy applications is sought. A bioenergy facility usually belongs to a wider system, therefore a holistic approach is adopted to define the location that optimizes the system-wide operational and investment costs. A hybrid optimization method is employed to overcome the limitations posed by the complexity of the optimization problem. The efficiency of the hybrid method is compared to a stochastic (genetic algorithms) and an exact optimization method (Sequential Quadratic Programming). The results confirm that the hybrid optimization method proposed is the most efficient for the specific problem.
\end{abstract}

(c) 2009 Elsevier B.V. All rights reserved.

\section{Introduction}

Facility location has been a challenging field of research for many decades. Locating a facility is usually a long-term investment, since it is associated with high investment costs. Furthermore, the location decision may have a great impact on the operational cost of the entire system associated with the facility, as upstream and downstream logistical costs have to be taken into account for the operational lifetime of the facility. These issues make facility location a critical aspect of strategic planning for a broad spectrum of public and private firms (Owen and Daskin, 1998).

A large number of mathematical programming models have been developed to deal with a wide range of location problems and applications encountered in the business world and the public sector. Continuous models, network location models and mixed-integer programming models are extensively reviewed in Klose and Drexl (2005). A branch and bound algorithm has also been applied to deal with a non-linear uncapacitated facility location problem

\footnotetext{
* Corresponding author. Tel.: +30210 7722383; fax: +30210 7723571 .

E-mail address: arent@central.ntua.gr (A.A. Rentizelas).
}

(UFLP) with integer variables and concave site dependent costs (Dupont, 2008).

Heuristic models such as tabu search, simulated annealing and genetic algorithms have been also widely applied (Arostegui et al., 2006). Heuristics were first put forward in the context of location models by Cooper (1964) and Teitz and Bart (1968). Their usage has proliferated tremendously since then and the most prominent among them, according to ReVelle et al. (2008), are genetic algorithms and tabu search. Neural networks have also been used to deal with plant location problems (Vaithyanathan et al., 1996).

Methods required to deal with multiple objectives have also been developed, especially in case of semi-desirable (or semi-obnoxious) facilities, such as landfills. Those methods include stochastic multicriteria acceptability analysis with ordinal criteria (Lahdelma et al., 2002) and hub location problems (Eiselt, 2007). In addition, Yang et al. (2007) used a combination of a fuzzy multi-objective programming and a genetic algorithm to locate fire stations, whereas Yapicioglu et al. (2006) applied biobjective particle swarm optimization to deal with the semi-desirable location problem. A combination of the analytic hierarchy process (AHP) with Goal Programming has been presented to tackle the global facility 
location-allocation problem (Badri, 1999). The interested reader may refer to Sahin and Sural (2007) for a thorough review on hierarchical facility location models and on ReVelle et al. (2008) for an annotated bibliography of recent papers in discrete location theory and modeling, related to: (1) the median and plant location models and (2) the center and covering models.

The various facility location models developed may be broadly classified, according to Owen and Daskin (1998) in:

- static and deterministic,

- dynamic, and

- stochastic.

The work presented here may be considered to lay in the static and deterministic category of facility location models. This type of problems can generally be further categorized to the most common fundamental areas of:

- median problems, where minimization of the average travel distance to or from the facility is the objective,

- covering problems, where minimum cost for a specific coverage level or maximum coverage for fixed number of facilities is required, and

- center problems (minimax), where minimization of the maximum distance between any demand and its nearest facility is sought.

\section{The biomass energy exploitation supply chain}

A typical biomass supply chain is comprised of several discrete processes. These processes may include ground preparation and planting, cultivation, harvesting, handling, storage, in-field/forest transportation, road transportation and utilization of the fuel at the power station.

Considering the typical locations of biomass fuel sources (i.e. in farms or forests) the transport infrastructure is usually such that road transport will be the only potential mode for collection of the fuel. Other factors that favor the use of road transport include the relatively short distances over which the fuel is transported and the greater flexibility that road transport can offer in comparison with other modes. Other transportation means, such as ship or train may be considered when long distance biomass transport is examined. However, this is not the case in this work, where emphasis is placed on locally existing biomass types.

In the present work, a relatively simple but typical biomass supply chain design has been adopted. The requirement of developing a generic supply chain model for examining several biomass types and also the multibiomass approach, including any combination of biomass types, led to the generic modeling of the supply chain design that is shown in Fig. 1.

The point of reference in the biomass-to-energy supply chain is the power plant. The upstream supply chain, concerns the biomass collection from the fields, its loading on to the transportation vehicles and its sub-

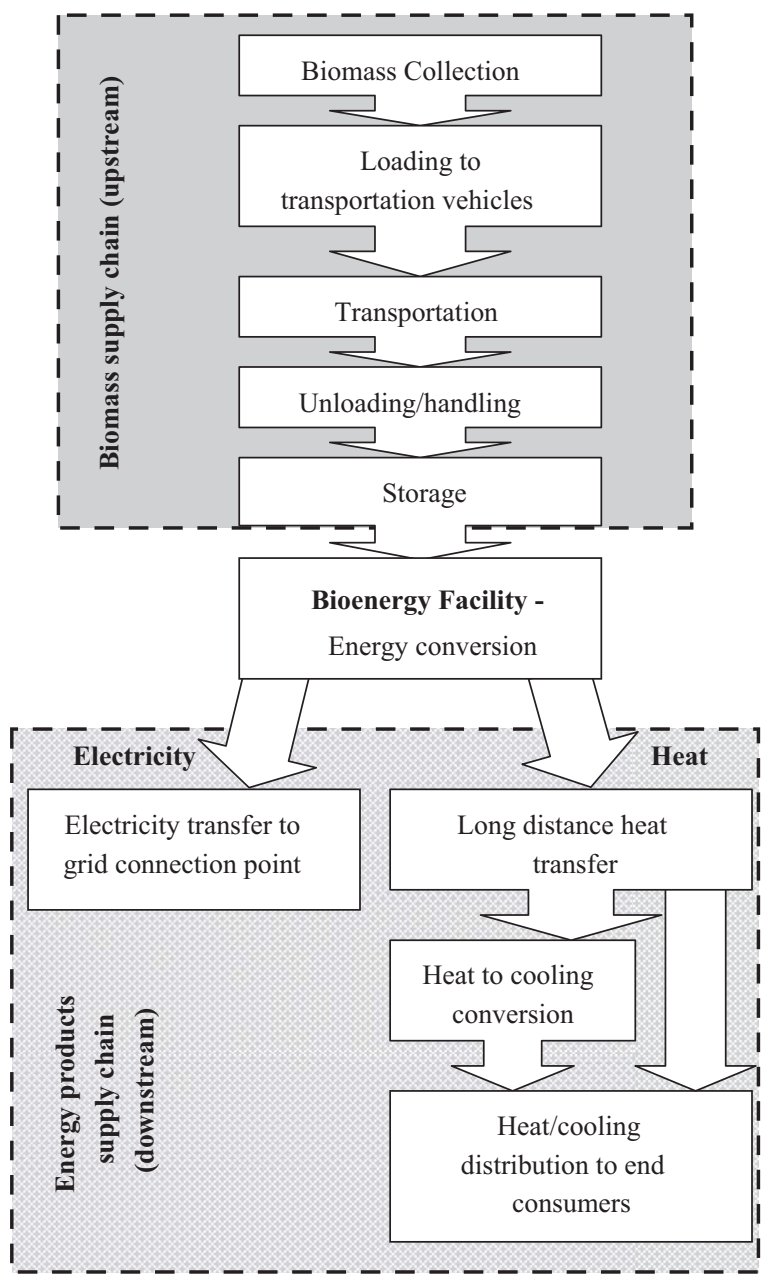

Fig. 1. The biomass energy exploitation supply chain.

sequent transportation to the storage space. The storage facility is considered to lay by the power plant, in order to remove a second transportation link that would be required in case of intermediate storage locations as well as to free the fields for the potential farming operations and land preparation, which would be obstructed if onfield biomass storage was chosen. The transportation vehicles unload the biomass at the storage yard and loaders undertake the handling and positioning of the biomass for storage. Then, biomass is fed into the power plant as required. The power plant usually generates electricity and heat.

The downstream supply chain concerns the two energy products. Electricity is transferred via the electricity transfer line to the grid connection point, where measuring devices for the electricity fed to the grid are installed. The heat generated is transferred via the main district heating pipeline to a location close to the district energy consumers, where a terminal point is installed. The terminal point contains heat exchangers and absorption chillers to convert heat into cooling when required, using heat as primary energy source, therefore enabling trigeneration. Trigeneration concerns the generation of three 
types of energy from the same facility, namely electricity, heat and cooling. The concept of trigeneration may be a solution for promoting district energy in relatively warm regions, like Greece and other south-European countries, as the operational time may be more than doubled. The same distribution network is used for district heating and cooling.

\section{The bioenergy facility location problem}

The bioenergy facility location problem is affected by a number of competing forces. As far as the upstream supply chain is concerned, the biomass availability and geographical dispersion are major factors affecting the potential location of the facility, due to their immediate effect on the mean travel distance, which determines the biomass transportation cost. However, mean travel distance is not the only parameter affecting the biomass supply chain cost. The time aspect has also an important effect, as the duration of the biomass collection period determines the number of collection, transportation and handling vehicles and equipment, the storage space as well as the labor requirements, thus ultimately affecting the biomass supply chain cost.

Moreover, a number of factors related to the downstream supply chain impact the total system cost. For example, the geographical positioning of the district heating and cooling consumers determines the length of the district energy transfer and distribution network. The length of the network does not only affect the investment cost, but the heat losses as well. Heat losses can be very significant in district energy systems of the size of a typical bioenergy facility, and can even render such an investment infeasible. Another factor affecting the facility location is the geographical positioning of the locally available grid connection points. The electricity transfer line length is thus determined, having a direct effect on the investment cost as well as the related electricity losses.

The typical decision concerning district heating facilities' location is to select a location as close as possible to the final district energy consumers in order to minimize the district heating network investment cost and heat losses. However, in the case of biomass-fueled district heating facilities, the abovementioned parameters of biomass geographical dispersion as well as the availability of electricity connection points need to be taken into account. Therefore, the decision problem is far more complicated when locally collected biomass is the primary fuel of the district energy facility compared to fossil-fueled facilities, thus requiring the application of optimization methods to determine the optimum facility location.

It should be mentioned that there are even more challenges connected to the biomass facility location problem. A crucial factor is the local communities' acceptance to the project, as the local opposition could prevent the construction of such a semi-desirable facility (Sinclair and Lofstedt, 2001; Upreti, 2004; Upreti and Van Der Horst, 2004). For this reason, it could be considered that the proximity of the facility to inhabited areas or areas of particular sensitivity is a factor determining the projects' acceptance.

It is apparent that the bioenergy facility location problem is a very particular case of the general facility location problem and is usually characterised by different objectives.

\subsection{Literature review on bioenergy facility location models}

The majority of models concerned with biomass energy exploitation are simulation models that investigate cases or scenarios of interest, assuming the location of the facility is pre-determined (Gallis, 1996; Nilsson and Hansson, 2001). However, a significant amount of research has also been devoted to optimization models that focus on the bioenergy facility location problem.

A linear programming (LP) optimization model has been utilized (Cundiff et al., 1997) to optimize a cost function including the biomass logistics activities between the on-farm storage locations and the centrally located power plant, construction and expansion costs of storage facilities, as well as the cost of violating storage capacity or lost revenue in case of biomass deficit. The authors consider the bioenergy facility location fixed and the LP model serves in choosing which farms among the existing ones will be chosen to supply biomass. A very detailed review concerning modeling tools for biomass supply chain and bioenergy conversion up to the year 1999 can be found in Mitchell (2000), where the author acknowledges the fact that most models tend to deal with only one aspect of the bioenergy system. However, most of the existing models are not concerned with sitting the facility, but with other aspects of the bioenergy system, such as technical issues on biomass conversion, technology selection, cost assessment, biomass potential estimation and logistics issues.

Several authors have also included the biomass-toenergy conversion facility in their biomass supply chain modeling efforts, examining the generation of electricity and/or heat. In the work of Tatsiopoulos and Tolis (2003) a detailed cotton-stalk supply chain model that employs linear programming (LP) optimization for the biomass delivery scheduling was presented. This model was applied for centralized (electricity only) and decentralized combined heat and power ( $\mathrm{CHP}$ ) power plant scenarios. The bioenergy facility is located in the center of the region examined for the centralized electricity scenario and LP is used to optimize the locations from which biomass is procured. For the decentralized scenario, the location of bioenergy facilities is determined indirectly, by calculating the optimum number of facilities and locating them uniformly on a grid. A GIS-based model to locate a bioenergy conversion facility based on optimum exploitation of available biomass potential was developed by Voivontas et al. (2001). In Papadopoulos and Katsigiannis (2002), the focus is mainly on sitting the bioenergy facility to reduce the biomass logistics costs, and more specifically, on eliminating biomass warehousing needs by performing a two-stage optimization: firstly, the CHP 
power plant location is determined to minimize the transportation distance and secondly, dynamic programming optimization is employed to identify the optimum biomass fuel mix.

None of the abovementioned models is designed to tackle the most practical problem, which concerns satisfying a currently existing energy demand (electricity and/or heat). Rather, these models mostly aim at determining (and some of them optimizing) the cost of biomass logistics and its energy conversion, while at the same time assuming that the energy generated will be exploited. Nevertheless, this assumption is very optimistic in real life conditions, where it is extremely difficult to find an existing heat or electricity demand that would perfectly match the economically interesting biomass potential calculated by these models.

Few models that focus on satisfying specific energy requirements have been developed, one of them presented in Nilsson (1999), where a biomass supply chain of two fuels (straw and reed canary grass) is simulated for use in district heating applications. The bioenergy facility location is defined by the model user; however, the intermediate storage locations are calculated by the simulation model. A similar approach, but only for one biomass type, was adopted in Nagel (2000) to determine an economic energy supply structure, covering existing heating demand with district heating network. The problem was formulated as a MILP optimization using a dynamic evaluation of economic efficiency, and binary operators to determine whether to construct or not a district heating network, a heating plant or a co-generation plant. The locations of heating plants were chosen among four pre-defined potential locations. Finally, a combination of GIS, mathematical modeling and optimization for energy supply at a regional level from forest biomass was presented by Freppaz et al. (2004). The system in question attempts to partially satisfy locally existing heat and electricity needs. The model developed employs GIS to calculate the transportation cost from all potential biomass collection points to all potential identified CHP plant locations. Then, optimization is performed regarding the optimal sizing of the power plant (defining which kind of energy to produce for the specific area), and biomass collection and harvesting scheduling.

It is therefore apparent that the biomass energy exploitation literature usually determines the location of the facility by various means other than optimization methods. In some cases optimization is employed to choose the final location among candidate identified locations. In this work, a wide geographical region around the energy consumers' location has been considered for the facility establishment, excluding regions defined by the constraints of the problem. A major difference between this work and the models presented in the literature is that the model developed aims at maximizing the total system financial yield while at the same time defining design and operational characteristics of the system including the facility location in question. Therefore, locating the facility is only one of the tasks the model has to perform. As a matter of fact, the model is intended to constitute a decision support system (DSS) for a potential investor, providing him with insightful information as well as suggestions on key issues such as the optimum size of the facility, the fuel source(s), the amount of each fuel type to be procured and last but not least the location of the facility. The suggested values for the variables of the model are derived by applying the concept of total system financial yield maximization. Consequently, a holistic optimization approach has been adopted for the entire system. It is obvious that in the case examined, the ultimate target is not the minimization of travel distance or time, nor the accomplishment of a specific coverage level, the maximum possible coverage level or the minimization of the maximum distance, which are usually the aims in facility location problems. On the contrary, a wider perspective of maximizing the total system yield is espoused.

\section{Optimization model}

The model presented in this work simulates and optimizes the operation of a system comprising of the biomass supply chain, the bioenergy conversion plant and the district heating and cooling (DHC) network that will supply the final customers with the energy products they require. The decision maker may decide which of the locally available biomass types will be included for consideration, as the model is parametric. The ultimate objective of the whole system simulation and optimization is to fully satisfy the thermal and cooling demand in the financially most efficient manner. Therefore, the system will operate at a heat-match mode. The facility comprises of a base-load co-generation module (CHP) and a biomass boiler for peak-load heat generation. Heat generated by the CHP unit and the biomass boiler may be used for heating purposes or it may be transformed to cooling using absorption chillers. The electricity generated will be sold at the national grid at prices that are determined by the Greek Regulatory Authority for Energy (RAE). The technology used for biomass-to-energy conversion may also vary, according to the decision makers' choice, as long as it is suitable for the biomass types selected and for CHP generation. In the case study, biomass gasification has been examined. The following notations are used for the mathematical formulation of the model (Table 1 ).

\subsection{Objective function}

The objective function to be maximized is the Net Present Value (NPV) of the investment for the project's lifetime. All the elements of the system are included in the investment analysis, i.e. the power plant, the supply chain of the biomass, the district heating and cooling (district energy) network with the connection to the customers, as well as the electricity transmission line and connection to the grid. All operational costs are also taken into account. NPV was chosen not only because it is the most frequently used investment appraisal criterion in co-generation plant investments (Biezma and San Cristobal, 2006), but also as 
Table 1

Notations.

\begin{tabular}{|c|c|c|}
\hline Sets and indices & & Description \\
\hline$i$ & $i=1 \ldots n$ & Biomass type \\
\hline$t$ & $t=1 \ldots T$ & Time period \\
\hline$l$ & $l=1 \ldots L$ & Distance class from power plant \\
\hline Variables & Units & Description \\
\hline$B_{i l}$ & tn wet biom. & Annual amount of the $i$ th biomass type to be procured from distance class $l$ \\
\hline Pmh & $\mathrm{kW}$ & Thermal capacity of the base-load CHP plant \\
\hline$P p$ & $\mathrm{~kW}$ & Thermal capacity of the peak-load biomass boiler \\
\hline$V_{0}$ & $\mathrm{~m}^{3}$ & Initial annual biomass inventory \\
\hline$V_{t}$ & $\mathrm{~m}^{3}$ & Biomass inventory in period $t$ \\
\hline$X p$ & $\mathrm{Km}$ & Longitude of bioenergy facility (geographical coordinate) \\
\hline$Y p$ & $\mathrm{Km}$ & Latitude of bioenergy facility (geographical coordinate) \\
\hline Parameters & Units & Description \\
\hline Ac & $€$ & Chilling equipment $\mathrm{O \& M}$ annual cost \\
\hline Ad & $€$ & District heating O\&M annual cost \\
\hline Aet & $€$ & Electricity transmission line O\&M annual cost \\
\hline$C b_{i}$ & $€ /$ tn wet & Purchasing and loading cost of biomass type $i$ \\
\hline$C c$ & $€ / M W h$ & Selling price of cooling \\
\hline Cch & $€ / \mathrm{kW}$ & Chiller specific investment cost \\
\hline $\mathrm{C}_{\mathrm{CO}_{2}}$ & $€ / \operatorname{tn} \mathrm{CO}_{2}$ eq. & Market price of a ton $\mathrm{CO}_{2}$ equivalent \\
\hline$C d$ & $€ / \mathrm{m}$ & Main district heating pipeline specific investment cost \\
\hline$C d n$ & $€$ & Distribution network \& connection cost per district energy customer \\
\hline $\mathrm{Ce}$ & $€ / \mathrm{MWh}_{\mathrm{el}}$ & Selling price of electrical energy \\
\hline Cetf & $€$ & Fixed investment cost of electricity transmission line \\
\hline Cetv & $€ / \mathrm{km}$ & Variable investment cost of electricity transmission line \\
\hline $\mathrm{Ch}$ & $€ / \mathrm{MWh}$ & Selling price of heat \\
\hline $\mathrm{Cm}$ & $€ / \mathrm{kW}_{\mathrm{el}}$ & Specific investment cost of base-load unit \\
\hline$C p$ & $€ / \mathrm{kW}$ & Specific investment cost of peak-load unit \\
\hline $\mathrm{Ctd}_{i}$ & $€ /(\mathrm{km} \mathrm{tn})$ & Distance-specific transportation cost of biomass $i$ \\
\hline$C t t_{i}$ & $€ /(\mathrm{htn})$ & Time-specific transportation cost of biomass $i$ \\
\hline$C v w$ & $€$ & Annual variable warehousing cost (e.g. salaries, handling) \\
\hline$C w$ & $€ / \mathrm{m}^{2}$ & Warehouse specific investment cost \\
\hline$D f$ & - & Discounting coefficient \\
\hline$D_{l}$ & $\mathrm{~km}$ & Biomass transportation trip distance for class $l$ \\
\hline$d m$ & $\mathrm{~kg} / \mathrm{m}^{3}$ & Mean biomass density \\
\hline$E c_{t}$ & MWh & Cooling generated in period $t$ \\
\hline Eec & $\mathrm{MWh}_{\mathrm{el}}$ & Electricity consumed in absorption chilling annually \\
\hline$E e_{t}$ & $\mathrm{MWh}_{\mathrm{el}}$ & Electricity generated in period $t$ \\
\hline$E h_{t}$ & MWh & Heat generated in period $t$ \\
\hline$E m h_{t}$ & MWh & Heat generated from the base-load CHP plant in period $t$ \\
\hline$E p h_{t}$ & MWh & Heat generated from the peak-load biomass boiler in period $t$ \\
\hline Gc & $\operatorname{tn} \mathrm{CO}_{2}$ eq. & Greenhouse gas (GHG) emissions reduction due to renewable cooling generation \\
\hline Ge & tn $\mathrm{CO}_{2}$ eq. & GHG emissions reduction due to renewable electricity generation \\
\hline$G t$ & tn $\mathrm{CO}_{2}$ eq. & GHG emissions reduction due to renewable heat generation \\
\hline$H$ & $\mathrm{~m}$ & Height of warehouse \\
\hline$i$ & $\%$ & Interest rate \\
\hline Il & $€$ & Warehousing equipment and loaders investment cost \\
\hline Ld & $\mathrm{m}$ & Length of the main district heating pipeline \\
\hline Le & $\mathrm{km}$ & Length of the electricity transmission line \\
\hline$L H V m$ & $\mathrm{~kJ} / \mathrm{kg}$ & Mean lower heating value of biomass \\
\hline Ls & $\mathrm{km}$ & Safety distance from heat \& cooling consumers \\
\hline$N$ & years & Investment lifetime \\
\hline$N d$ & - & Number of district energy customers \\
\hline ne & $\%$ & Electricity transmission losses \\
\hline$n m$ & $\%$ & Total efficiency factor of base-load unit \\
\hline$n p$ & $\%$ & Total efficiency factor of peak-load unit \\
\hline $\mathrm{Om}$ & $\%$ & Base-load unit annual O\&M cost (\% of investment cost) \\
\hline Op & $\%$ & Peak-load unit annual O\&M cost (\% of investment cost) \\
\hline Ow & $€ / \mathrm{m}^{2}$ & Warehouse annual operation \& maintenance (O\&M) cost \\
\hline Pch & $\mathrm{kW}$ & Chillers installed capacity \\
\hline PHR & - & Power-to-Heat ratio \\
\hline Pme & $\mathrm{kW}_{\mathrm{el}}$ & Electrical capacity of the base-load CHP unit (=Pmh*PHR) \\
\hline$P h d_{t}$ & $\mathrm{~kW}$ & Mean monthly equivalent thermal demand of customers \\
\hline Sc & $\%$ & Public subsidy on investment for chilling equipment \\
\hline Set & $\%$ & Public subsidy on investment for electricity transmission \\
\hline$S p$ & $\%$ & Public subsidy on investment for power plant \\
\hline$T_{20}$ & days & 20-day period \\
\hline$T_{1}$ & $\mathrm{~h}$ & return trip time for distance class $l$ \\
\hline$W$ & $\mathrm{~m}^{2}$ & Warehouse area \\
\hline
\end{tabular}


Table 1 (continued)

\begin{tabular}{lll}
\hline Sets and indices & & Description \\
\hline$X b_{1}$ & $\mathrm{~km}$ & Longitude of biomass fields belonging to l distance class \\
$X d$ & $\mathrm{~km}$ & Longitude of heat \& cooling consumers \\
$X e$ & $\mathrm{~km}$ & Longitude of electricity connection point to grid \\
$Y b_{1}$ & $\mathrm{~km}$ & Latitude of biomass fields belonging to l distance class \\
$Y d$ & $\mathrm{~km}$ & Latitude of heat \& cooling consumers \\
$Y e$ & $\mathrm{~km}$ & Latitude of electricity connection point to grid \\
$\rho$ & $\%$ & Inflation rate \\
\hline
\end{tabular}

it is considered theoretically superior to other criteria (Zimmerman, 1997). The NPV function to be maximized is

$$
\begin{aligned}
\operatorname{Max} N P V= & {\left[C e(1-n e)\left(\sum_{t} E e_{t}-E e c\right)+C h \sum_{t} E h_{t}\right.} \\
& \left.+C c \sum_{t} E c_{t}+C \operatorname{co} 2(G e+G h+G c)\right] D f \\
& -[(C w W+I l)+(C m P m h P H R+C p P p)(1-S p) \\
& +(C e t v L e+C e t f)(1-S e t)+C d L d \\
& +C d n N d+C c h P c h(1-S c)] \\
& -\left[\sum_{i} \sum_{l} B_{i l} C b_{i}\right. \\
& +\sum_{i} \sum_{l} B_{i l}\left(C t d_{i} D_{l}+C t t_{i} T_{l}\right)+O w W \\
& +C v w+O m C m P m h P H R+O p C p P p+A e t \\
& +A d+A c] D f
\end{aligned}
$$

$L e=\sqrt{(X p-X e)^{2}+(Y p-Y e)^{2}}$

$L d=\sqrt{(X p-X d)^{2}+(Y p-Y d)^{2}}$

$D_{l}=\sqrt{2\left[\left(X p-X b_{l}\right)^{2}+\left(Y p-Y b_{l}\right)^{2}\right]}$

$W=\frac{1,1}{H} \max V_{t}$

where in (1a) the first group of terms in brackets refers to the revenue streams of the investment, the second group to the investment costs and the third group to the operational costs. All the annual monetary amounts are multiplied by an appropriate discounting coefficient $D f$, which turns them into present values, assuming that the annual amounts will follow the inflation rate and that the inflation rate will remain fixed for the investment's lifetime:

$D f=\frac{1-\left[1+\frac{(i-\rho)}{(1+\rho)}\right]^{-N}}{i-\rho}$

It should be noted that the objective function calculates the NPV before taxes.

\subsection{Optimization variables}

Two of the optimization problem variables are the longitude and latitude of the facility location, which determine the exact location of the power plant. However, there are several more independent variables that describe the system and are determined by the optimization method. The independent variables of the optimization problem are the following:

- Xp\&्Yp: The optimum location (geographical coordinates) to construct the bioenergy facility.

- Pmh: The thermal capacity of the base-load CHP plant. The electrical capacity of the plant (Pme) is assumed to be proportional to the thermal capacity.

- Pp: The thermal capacity of the peak-load biomass boiler.

- $B_{i}$ : The total amount of the $i$ th biomass type to be procured each year.

- $V_{0}$ : The initial annual biomass inventory. This variable is necessary, as the calculations are based on a rolling horizon framework, similarly to Cundiff et al. (1997).

- $V_{t}$ : Monthly biomass inventory.

\subsection{Constraints}

Several constraints have been introduced in the mathematical formulation of the problem.

\subsubsection{Energy demand constraints}

The biomass power plant must have enough capacity installed to satisfy the thermal or cooling peak loads of the consumers:

$P m h+P p \geq \max P h d_{t}$

where $\max P h d_{t}$ is defined as the maximum thermal (or cooling) demand of the customers for a predefined confidence level, converted into equivalent heat demand. This constraint ensures that the heat produced each time period by the base-load CHP unit and the peak-load boiler will satisfy the thermal or cooling energy demand of the DHC customers.

\subsubsection{Warehousing constraints}

There should be a biomass safety stock in the warehouse at any time to meet the energy needs of the customers for a certain timeframe. This is here assumed as the amount of biomass adequate for at least twenty days of full-load operation for both base- and peak-load units:

$V_{t} d m L H V m \geq\left(\frac{P m h(1+P H R)}{n m}+\frac{P p}{n p}\right) T_{20} \forall t$

Another constraint is introduced, due to the rolling horizon of the model: The finishing season stock $\left(V_{T}\right)$ 
must be at least as much as the starting season stock $\left(V_{0}\right)$. A case where $V_{T}$ would be larger that $V_{0}$ could be acceptable and have a physical meaning, as the difference could be interpreted as material loss. However, the application of optimization leads practically always to equal starting and ending period inventory:

$V_{T} \geq V_{0}$

\subsubsection{Legislation constraints}

There is a legislation requirement that a co-generation project may receive subsidy on investment only if at least a certain percent (presently 65\%) of the heat generated is actually exploited:

$\sum_{t} E h_{t} \geq 65 \% \sum_{t}\left(E m h_{t}+E p h_{t}\right)$

where $E h_{t}$, equivalent heat demand of district energy consumers during a specific time period $t\left(\mathrm{MW} \mathrm{h}_{\mathrm{th}}\right)$; $E m h_{t}$, heat generated from the CHP unit during a specific time period $t\left(\mathrm{MWh}_{\mathrm{th}}\right) ; E p h_{t}$, heat generated from the biomass peak-load boiler during a specific time period $t\left(\mathrm{MW} \mathrm{h}_{\mathrm{th}}\right)$

This constraint is active when the operational mode of the power plant is not heat-match. In the application examined here, the assumption of heat-match mode operation ensures that this constraint is always met, since the heat generated equals the heat demand.

\subsubsection{Social constraints}

Certain social or environmental conditions may prohibit the installation of the bioenergy conversion facility in some regions. More specifically, the biomass power plant should not be located very close to the DHC customers' location, which will probably be an inhabited area, due to potential local opposition (Upreti and Van der Horst, 2004; Upreti, 2004). In facility location literature there exists a specific problem category, named semi-obnoxious or semi-desirable facility location, for facilities such as garbage dump sites, airports and power plants (Brimberg and Juel, 1998), where usually a bi-objective or multiobjective problem has to be solved. In semi-obnoxious problems the two contradictious objectives of maximizing the distance from communities negatively affected and minimizing operational costs have to be met (Yapicioglu et al., 2006). In this case study the disservice generated by the biomass power plant has been treated as a constraint rather than developing a bi-objective model. It is therefore assumed that the bioenergy conversion facility must be located at least a safety distance (Ls) away from the customers' location $(X d, Y d)$, to avoid potential local opposition.

$(X p-X d)^{2}+(Y p-Y d)^{2} \geq L s^{2}$

\subsubsection{Logical constraints}

Certain logical constraints are introduced in the optimization problem. The independent variables are required to be non-negative and upper bounds are also defined for many of them. For example, the annual amount of each biomass type is bounded by the maximum available biomass quantity of this type ( $\left.\max B_{i l}\right)$ in the distance class $l$ under examination.

$0 \leq B_{i l} \leq \max B_{i l} \forall i, l$

The biomass boiler size has a lower bound equal to the minimum monthly heating and cooling demand of the final consumers, multiplied by a safety factor (equal here to $120 \%$ ). The rationale behind this bound is that the boiler should be able to serve fully the heat and cooling demand at least for the month with the minimum demand, in order to allow maintenance of the main CHP unit without disrupting the energy supply to the consumers:

$120 \% \min \mathrm{Phd}_{t} \leq \mathrm{Pp}$

where $P h d_{t}$, monthly equivalent heat demand of district energy consumers $\left(\mathrm{kW}_{\mathrm{th}}\right)$.

The longitude and latitude of the biomass power plant location have user-defined upper and lower bounds, as long distance district heating and cooling is inefficient:

$\min X p \leq X p \leq \max X p$

$\min Y p \leq Y p \leq \max Y p$

\subsection{Optimization}

Numerous optimization methods exist, as optimization is a huge field of operational research. Some of them are applicable only to specific types of problems, whereas others are generally applicable. However, even those "generic" optimization methods are usually more efficient when applied to specific kinds of optimization problems.

In the bioenergy supply chain literature, several optimization methods have been applied. Linear programming (LP), a method that has the advantage of simplicity and assurance of identifying the global optimum has been used in Cundiff et al. (1997) and Tatsiopoulos and Tolis (2003). These two models managed to retain linearity of the model by optimizing only the biomass supply chain and not the entire system. MILP has been used in one instance (Nagel, 2000) to include binary operators for investment decisions in the variables. Furthermore, dynamic programming has been used in Papadopoulos and Katsigiannis (2002) to identify the optimum fuel mix for a biomass CHP unit.

In the general facility location literature, various optimization methods have been widely applied. In many cases linear models are used (Appa and Giannikos, 1994). In some cases non-linear objective functions are approximated by piecewise linear functions to transform a nonlinear problem to linear, as in Aboolian et al. (2007). Lately, heuristics such as tabu search (TS), simulated annealing (SA) and genetic algorithms (GA) have been applied to the facility location problem (FLP) with good results (Arostegui et al., 2006).

\subsubsection{Optimization problem}

The optimization problem arising from the model presented in this paper is non-linear, as it aims at modeling and optimizing the entire bioenergy system, thus excluding LP from the candidate optimization methods. Non-linearity 
is introduced due to the modeling of economies of scale. Apart from non-linearity, the objective function of the optimization problem in question presents another challenge for the optimization method: it has several discontinuities, which are introduced by the analytical modeling of the biomass supply chain. The objective function is a piecewise continuous function, similar to the case of Nilsson (1999). For example, it is possible that for a very small difference in incoming biomass, one more loader will be required to handle the extra amount of biomass. Therefore the cost function will make a step. The number of the potential non-linearities has been found to be relatively small and their impact is also judged as mild. However, no one can ensure that this feature may not present a problem for an exact optimization method, if the region of a discontinuity happens to be evaluated. The specific characteristics of the optimization problem complicate the decision for selection of an optimization method that will be able to cope with the abovementioned challenges. Furthermore, the inherent inability of most of the currently existing non-linear optimization methods to ensure the identification of the global optimum of the problem has been a major concern.

In order to overcome the limitations of using a specific non-linear optimization method, a hybrid method is applied in the model. This means that firstly, one optimization method is employed to define a good solution to the problem. This solution is used as the starting point of the second optimization method that bears the task to enhance further the solution found at the first step.

\subsubsection{Optimization method}

The optimization method employed as the first step of the hybrid method is the genetic algorithms (GA). The GA method has been applied for a great variety of optimization problems and is based on the principles of genetics and natural selection. A GA allows a population composed of many individuals to evolve under specified selection rules to a state that maximizes the selected criteria (Haupt and Haupt, 2004). Some of the advantages of a GA include that it optimizes even non-linear, non-continuous and non-differentiable functions with continuous or discrete variables, it does not require derivative information, it simultaneously searches from a wide sampling of the cost surface, it deals with a large number of variables and it can find a relatively good solution in short time. Even more importantly, a GA may succeed in finding the global optimum due to the fact that the method evaluates simultaneously a large population instead of a single point for most non-heuristic optimization methods. Finally, a GA does not require a user-defined starting point for an efficient operation. These advantages are intriguing and can produce stunning results when traditional optimization approaches fall miserably (Haupt and Haupt, 2004).

A disadvantage of a GA is that, despite the fact that there is a good chance of finding a solution close to the global optimum, the method progresses very slowly after a certain point, especially for complex problems. Therefore, this method is not particularly efficient for local search.

The characteristics of GA have been confirmed by Arostegui et al. (2006), who compared the results of applying the heuristic optimization methods of TS, SA and GA for a number of typical FLP. The authors have concluded that GA outperform the other methods in some cases, but most importantly they provide a very good result at a shorter computational time compared to the other two methods in all problems examined. The authors also acknowledge that GA can extract more information from fewer solutions, and that their application in FLP results in an initially steep descent, which is slowed down significantly at the last stages of the optimization procedure. The hybrid method presented in this work aims at exploiting the initial steep descent of the GA method, while at the same time eliminating its disadvantage of slow progress at the last stages of optimization.

For this reason, an exact quasi-Newton optimization method is applied as the second step to define the optimum. This method was chosen to be Sequential Quadratic Programming (SQP), which solves a quadratic programming subproblem at each step. This type of continuous optimization method presents the advantage of very fast convergence with accuracy in optimum solution identification. Its disadvantage is mainly the fact that it may identify a local optimum instead of the global, and that the results may be disappointing if one does not use a good starting point. In many practical cases, as well as in the case described in this paper, finding a good starting point is actually impossible. Furthermore, this method requires continuous objective function, its first and second derivatives and constraints.

The hybrid optimization method applied in this model aims at retaining the advantages of each method while at the same time eliminating or significantly reducing their disadvantages and limitations. Having defined a good solution in the vicinity of the global optimum using the GA, the application of the SQP method with the GA optimum as its starting point may lead to identification of the global optimum with high accuracy in a significantly reduced computational time. The possibility of SQP hitting a discontinuity point of the objective function is dramatically reduced, since this method is used for local search. The proposed hybrid optimization method utilizes each optimization method for the tasks it can perform most efficiently.

\subsubsection{Optimization method parameters}

The parameters of the genetic algorithms have been selected with the main objective of widely searching the solution space. Accordingly, a rather large population size has been selected (200 genomes), to facilitate the identification of the global optimum region. The initial population is created randomly, subject to a range which is determined by the constraints of the problem. The constraints have been modeled as linearly increasing penalty functions, as it has been found more efficient than any other type of penalty function. The scaling of the chromosomes is performed using a simple ranking function, which scales the raw scores based only on the rank of each individual, instead of its score, therefore removing the effects of the spread of the raw scores. The 5 best chromosomes of each generation are considered as elite and pass to the next generation intact. The parents are selected using a stochastic uniform function. The algorithm 
of the selection function moves along a line, which consists of sections proportional to each parents' scaled value, in steps of equal sizes. The sections on which the algorithm lands denote the parents selected. An adaptive feasible mutation function has been employed, which randomly generates directions that are adaptive with respect to the last successful or unsuccessful generation. A step length is chosen along each direction so that the constraints and bounds are satisfied. A high value for the mutation factor (0.35) has been chosen to examine a significant number of new solutions in each generation and to deter early convergence of the first step of the hybrid optimization method. Finally, a scattered crossover function has been chosen, which creates a random binary vector and selects the genes where the vector has a value of 1 from the first parent, and the genes where the vector has a value of 0 from the second parent, and combines the genes to form the child. The high mutation factor results in a relatively low crossover factor of 0.65 .

The SQP method employed does not use penalty functions, but models the optimization problem as a constrained non-linear optimization problem. The optimum solution is reached when first order optimality conditions are satisfied to a specified tolerance level. There is also a limit of maximum iterations and function evaluations number, for the case that the method fails to converge to a solution.

\section{Case study-system description}

The case study concerns the potential establishment of a biomass trigeneration plant in the district of Thessaly, Greece. Thessaly is the largest plain in Greece, and there exist many types of cultivations, therefore making the region an ideal candidate to apply the concept of multiple biomass exploitation. The types of biomass examined include several types of agricultural residues, such as wheat straw, maize, cotton stalks and prunings from olive trees and almond trees. These biomass types have been characterized as dominant in the region, using Pareto analysis, and all of them are considered as potential fuel sources for the power plant. The basic characteristics of the biomass sources considered in the analysis are presented in Table A1 of Appendix A. A local community is considered to be the heat and cooling customer. A total of 500 households, currently using heating oil for space heating and electrical heat pumps for cooling, are assumed to be connected to the district energy network. The facility will operate on heat-match mode. Real statistical biomass availability data has been used, and the amount of biomass existing in the region has been found to be significantly larger compared to the needs of the small to medium-size bioenergy facility under investigation. The investor could either be a private entity or a regional authority. The main case study input data are presented in Table A2 of Appendix A.

The main revenue sources of the power plant under consideration are electricity sales to the national grid, heat and cooling supply to the customers via a district heating network as well as emissions reduction units' (ERU's) trading. The electricity produced will be sold directly to the national grid, at prices determined by the Greek energy authority. The price of heat is assumed to be a fixed percentage of the cost of heat obtained by using oil whereas the price of cooling is a fixed percentage of the cost of cooling obtained by electrical compression chillers.

\section{Results}

The bioenergy facility location is of crucial importance for the financial yield of the investment. A map of the geographical region around the location of the district energy consumers is shown in Fig. 2. The contour lines represent the potential facility locations with the same value of NPV for the investment. The values of the contour lines are in million Euros. Cost contour lines are an interesting method of obtaining insight to the behavior of the objective function and to alternative facility locations (Nanthavanij and Asadathorn, 1999). This map was created by eliminating the bioenergy facilities' longitude and latitude variables from the optimization model and by forcing the model to optimize the rest of the variables for the geographical coordinates of each potential location on a grid. The shaded circle around the energy consumers' location signifies the proximity constraint, denoting the area around the village where the facility should not be established to avoid potential public opposition. The red " $x$ " sign shows the bioenergy facility location suggested by the hybrid optimization model and the blue solid lines show the geographical coordinates of this location.

Some interesting comments may be made on Fig. 2. First of all, it can be seen that the hybrid optimization method proposes a location on the borders of the proximity constraint, which means an effort has been made to minimize the district energy network investment cost, heat losses and operational cost. Furthermore, the proposed location facility is at the south-west of the village, where the NPV value is higher due to the higher biomass availability. The map of Fig. 2 may be very useful to the potential investors, as it reveals alternative locations for the facility, with only a minor effect on the financial yield of the project. This fact may significantly reduce the risk inherent with site selection, as an alternative location may be easily chosen, thus overcoming any problems encountered with the location originally selected by the optimization method.

As far as the operation of the hybrid optimization method is concerned, a snapshot of the operation of the GA step is shown in Fig. 3. The blue colored crosses represent the mean value of the objective function and the black points the best value for each generation.

It should be noted here that the objective function is expressed in such a way that the optimum value (maximum NPV) is obtained by minimizing the objective function. Fig. 3 shows that the mean value of the objective function increases at the first generations, while at the same time the best value significantly reduces. This fact reveals the GA tendency to search widely in the search space. It could also be inferred that the wide search leads ultimately to identification of improved genomes, despite the increase in the mean value of the population. 


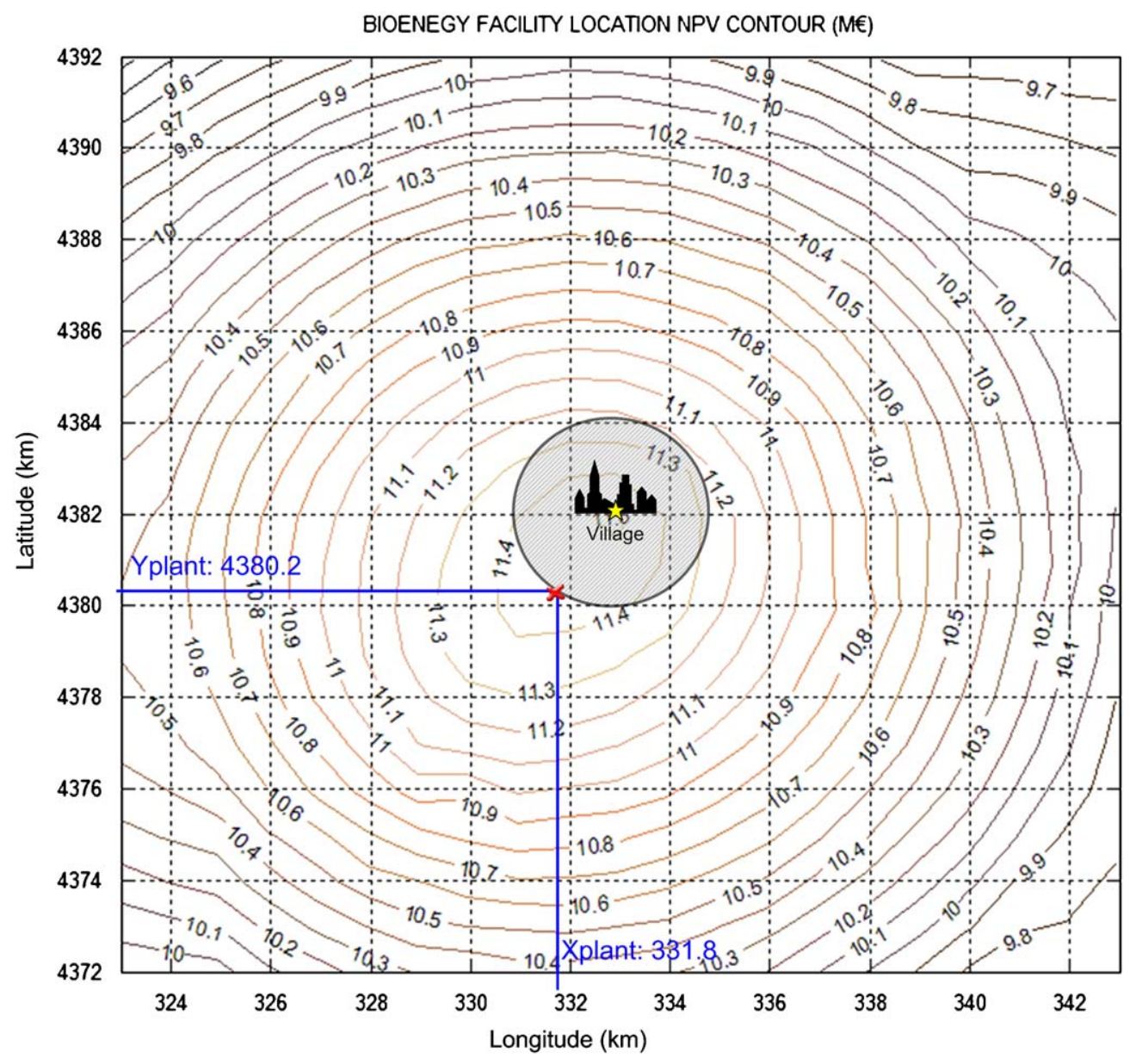

Fig. 2. Bioenergy facility NPV contour map (contour values in million Euros).

It is interesting to note that the mean value of the population increases in several instances, when the mean value is approximating its best value. This phenomenon is due to the large mutation factor used for the specific application. As generations pass by, the periodical increases of the mean value tend to have smaller intensity.

An attempt has been made to verify the merits of the hybrid optimization method applied for the specific problem, by comparing the results with the two optimization methods that are actually its constituents: SQP and GA. The three methods have been applied independently for the same optimization problem and the optimum solution determined by each one is presented in Table 2 . The solution presented is the best determined after ten runs with different starting points/populations, in order to avoid a single potential case where the algorithm may diverge and provide meaningless results.

It can be inferred from Table 2 that the result of the hybrid optimization method is better than the one of the GA or SQP methods. The SQP method converges to a solution with an objective function value $0.13 \%$ less than to the one determined by the hybrid method, while the GA method falls short by $0.61 \%$. The SQP method requires significantly less computational time compared to the other two methods. However, the time required is reasonable in all cases.
It is impressive that the solutions determined by the hybrid and the GA methods present many similarities, whereas the SQP method converges to a solution with major differences in the energy generation modules' nominal power and the geographical location of the power plant. This fact implies potential trapping of the SQP method to a local optimum, which is a major argument for the development of the hybrid method. On the other hand, it is obvious that the GA method is unable to perform local search, as it identifies the global optimum neighborhood, but not with high accuracy.

A very critical characteristic of an optimization method is its robustness. Since the optimization problem entails a piecewise continuous objective function, it is very important to examine the methods' robustness, namely how often the optimum solution is identified for a large number of applications and how far lie the solutions found from the real optimum of the problem. For this reason, each optimization method has been applied a hundred times with different starting points/ populations. The results are shown in Fig. 4, where the solutions are classified according to their deviation from the optimum solution found. It should be noted that in Fig. 4, the scale of the $X$ axis is custom and not linear, focusing mainly on solutions very close to the real optimum. 


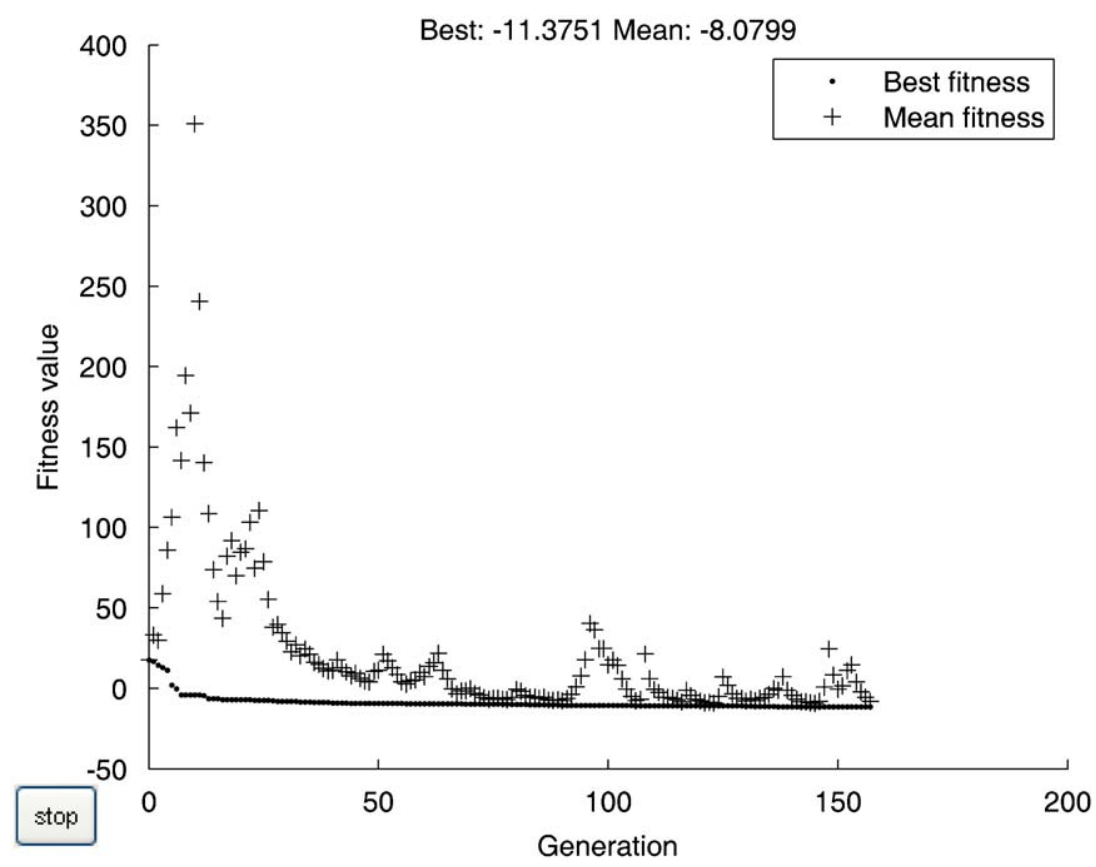

Fig. 3. GA operation snapshot.

Table 2

Optimum solution for various optimization methods.

\begin{tabular}{|c|c|c|c|}
\hline & Hybrid method & GA & SQP \\
\hline NPV $(\mathbf{M} €)$ & 11,461 & 11,392 & 11,446 \\
\hline Computational time & $15 \mathrm{~min}$ & $14 \mathrm{~min}$ & $15 \mathrm{~s}$ \\
\hline \multicolumn{4}{|l|}{ Optimization variables } \\
\hline CHP thermal power $\left(\mathrm{MW}_{\mathrm{th}}\right)$ & 4126.7 & 4187.6 & 3677.6 \\
\hline Biomass boiler thermal power $\left(\mathrm{MW}_{\mathrm{th}}\right)$ & 500 & 507.8 & 952.4 \\
\hline Biomass 1 Wheat straw $\left(\operatorname{tyr}^{-1}\right)$ & 2013.7 & 2375.1 & 2345.2 \\
\hline Biomass 2 Corn stalks $\left(\mathrm{tyr}^{-1}\right)$ & - & 102.6 & - \\
\hline Biomass 3 Cotton stalks $\left(\mathrm{t} \mathrm{yr}^{-1}\right)$ & 4424.2 & 4000.1 & 3736 \\
\hline Biomass 4 Olive tree prun. $\left(\mathrm{tyr}^{-1}\right)$ & - & 13.1 & - \\
\hline Biomass 5 Almond tree prun. (t $\left.\mathrm{yr}^{-1}\right)$ & 4387.5 & 4469.3 & 4617.9 \\
\hline Initial biomass inventory $\left(\mathrm{m}^{3}\right)$ & 4225 & 4359.5 & 4106.2 \\
\hline Plant longitude (km) & 331.57 & 330.74 & 334.24 \\
\hline Plant latitude (km) & 4380.46 & 4379.73 & 4380.41 \\
\hline
\end{tabular}

The global optimum solution found with the hybrid method for a slightly simplified problem has been compared to the real optimum of the problem, which has been calculated using the complete enumeration method, with very significant computational time cost. It has been found that the hybrid method can achieve a solution with a difference of about $0,01 \%$ to the real optimum found by the complete enumeration.

In Fig. 4 one may notice that the solutions of the hybrid method have a larger concentration around the real optimum, as compared to GA or SQP methods. More specifically, $71 \%$ of the solutions found by the hybrid method have an objective function value more than $99 \%$ of the real optimum one, whereas SQP achieves this only in $44 \%$ of the cases and GA in $33 \%$. Similarly, when referring to objective function values over $98 \%$ of the real optimum solution, the hybrid method has passed the threshold in $87 \%$ of the cases, SQP in $54 \%$ and GA in $40 \%$.
Despite the high concentration of the solutions found around the real optimum for the hybrid method, it is interesting to note that the mean solution found by the SQP is slightly better than the hybrid method, and the standard deviation of the solutions found is surprisingly smaller (Table 3). The hybrid method is affected by the GA application as a first step, and GA diverge in almost $5 \%$ of the runs, where the term "divergence" is used here to signify a solution found with objective function value less than $60 \%$ of the real optimum. The hybrid method performs slightly better, but still it diverges in $4 \%$ of the runs. These few cases of divergence lead to the increased standard deviation of the hybrid method. In order to indulge further in this issue, the mean and standard deviation have been calculated again for the best $95 \%$ of the solutions found, in an attempt to remove the outliers (Table 4).

It is apparent from Table 4 that the hybrid method determines solutions with increased mean value and 


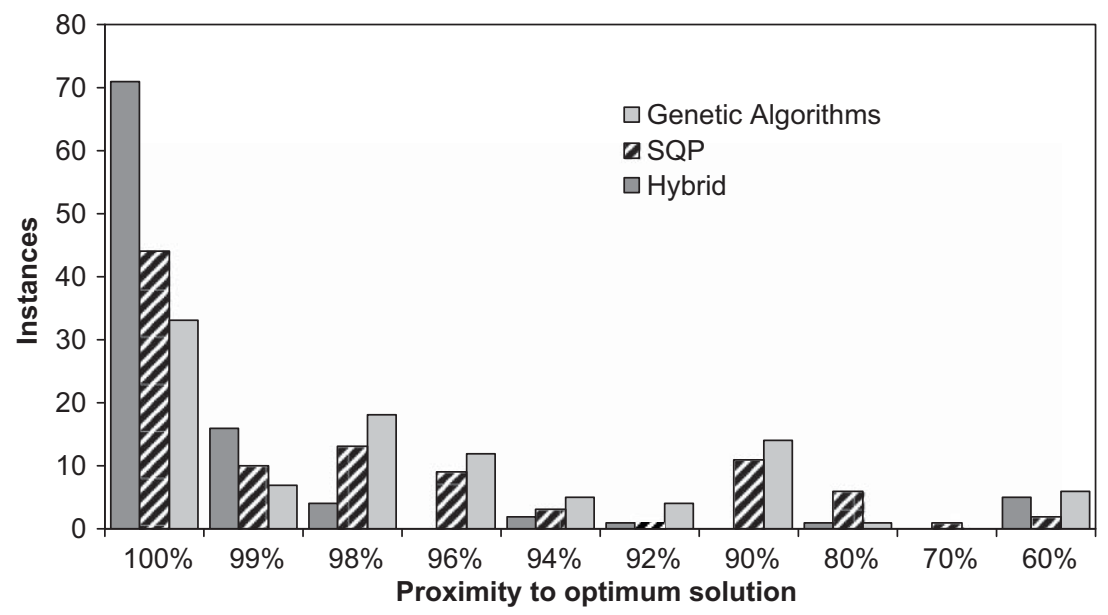

Fig. 4. Robustness of the GA, SQP and hybrid optimization methods.

Table 3

Mean and standard deviation for 100 runs.

\begin{tabular}{lll}
\hline Method & Mean $\left(\times \mathbf{1 0}^{\mathbf{3}}\right)$ & Standard deviation $\left(\times \mathbf{1 0}^{\mathbf{3}}\right)$ \\
\hline Hybrid & 10663.0 & 3072.2 \\
SQP & 10783.8 & 1369.1 \\
GA & 10127.9 & 3274.6 \\
\hline
\end{tabular}

Table 4

Mean and Standard Deviation for the best $95 \%$ of the solutions found.

\begin{tabular}{llc}
\hline Method & Mean $\left(\times \mathbf{1 0}^{\mathbf{3}}\right)$ & Standard deviation $\left(\times \mathbf{1 0}^{\mathbf{3}}\right)$ \\
\hline Hybrid & 11361.1 & 299.0 \\
SQP & 11026.0 & 681.9 \\
GA & 10798.1 & 1488.1 \\
\hline
\end{tabular}

significantly reduced standard deviation compared to the SQP method, when only the best $95 \%$ of the solutions are taken into account. Furthermore, GA performance is poorer than that of the SQP method, having more than twice the standard deviation of the SQP.

\section{Conclusions}

In this work, a biomass energy exploitation facility location model has been presented. The methodology has been applied to a case study, in order to optimize the location as well as design and operational parameters of a biomass trigeneration plant in Greece, serving specific energy needs. The bioenergy facility location problem has been modeled as a non-linear optimization problem with the investment yield serving as the objective function. Furthermore, a number of constraints have been introduced. The concept of system-wide optimization when selecting the location of such a facility is innovative for the specific field of applications. The optimization concerns a number of decision variables for the investor, apart from the location of the facility, such as the size of the facility and the annual amount of each biomass type available to use for energy generation.
A hybrid optimization method has been developed to allow the application of this concept, which is a novelty for the biomass supply chain energy exploitation research. The optimization method proposed appears to be competent in handling the complexity and the peculiarities of the bioenergy facility location problem. More specifically, the hybrid optimization method not only provides solutions of higher quality compared to the GA and SQP, but it also provides them with increased reliability for the specific problem, which is a very significant characteristic for an optimization method. This method actually appears to retain the virtues of both its constituent methods, while significantly mitigating their limitations. However, one should perform more than one runs of the hybrid method, to avoid the small possibility of the method diverging. A proposed number of ten runs will provide a solution very close to the real optimum one with very high reliability, and within reasonable computational time.

Furthermore, the NPV contour map presented for the case study constitutes a major decision support tool for the potential investors, as it provides information on alternative locations for establishing the facility with only a minor effect on the investment yield. The information provided may significantly reduce the risk of inhibiting the investment due to unexpected problems or implications encountered for the optimum location defined by the hybrid optimization model. In practical cases, this kind of information is invaluable for the investors, especially when a semi-desirable facility is concerned.

Finally, it should be noted that the future plans of the authors include a number of different case study applications of the hybrid optimization method to come up with even more detailed information on the performance of this method.

\section{Appendix A}

The basic characteristics of the biomass sources considered in the analysis are presented in Table A1. The main case study input data are presented in Table A2. 
Table A1

Characteristics of five dominant biomass types in the case study area considered.

\begin{tabular}{|c|c|c|c|c|c|}
\hline & 1. Wheat straw & 2. Corn stalks & 3. Cotton stalks & 4. Olive tree prunings & 5. Almond tree prunings \\
\hline Residue yield ( $\mathrm{t} / \mathrm{ha})^{\mathrm{a}}$ & 2.97 & 7.17 & 5.47 & 2.82 & 6.21 \\
\hline Residue availability factor (\%) & 15 & 30 & 70 & 90 & 90 \\
\hline Exploitable residue (t/ha) & 0.45 & 2.15 & 3.83 & 2.54 & 5.59 \\
\hline Moisture wet (\%) & 20 & 50 & 30 & 35 & 40 \\
\hline Higher heating value (MJ/dry kg) ${ }^{a, b}$ & 17.9 & 18.4 & 18.1 & 18.1 & 18.4 \\
\hline Availability period & July-August & November-December & Octobter-November & December-February & December-February \\
\hline Residue price ( $€ /$ ton wet $)^{c}$ & 50 & 20 & 20 & 30 & 30 \\
\hline
\end{tabular}

a Source: Voivontas et al. (2001).

b Source: Papadopoulos and Katsigiannis (2002).

c Residue price includes purchasing and loading cost, prices assumed.

Table A2

Main case study input data.

\begin{tabular}{|c|c|}
\hline Interest rate (\%) & 8 \\
\hline Inflation (\%) & 3 \\
\hline Investment lifetime (yr) & 20 \\
\hline Transportation \& handling equipment lifetime (yr) & 7 \\
\hline Subsidy on bioenergy facility investment (\%) & 40 \\
\hline Subsidy on DHC network \& equipment (\%) & 40 \\
\hline Subsidy on electricity transmission line (\%) & 0 \\
\hline Electricity selling price $(€ / \mathrm{MWh})$ & 68.42 \\
\hline Heat selling price $(€ / M W h)$ & 47.8 \\
\hline Cooling selling price $(€ / M W h)$ & 36 \\
\hline Oil price $(€ / \mathrm{kg})$ & 0.5 \\
\hline Electrical efficiency of CHP unit (\%) & 29 \\
\hline Total efficiency of CHP unit (\%) & 85 \\
\hline Thermal efficiency of biomass boiler (\%) & 80 \\
\hline COP of absorption chillers & 0.7 \\
\hline O\&M of CHP unit (\%inv. cost $\mathrm{yr}^{-1}$ ) & 7 \\
\hline O\&M of biomass boiler (\%inv. cost $\mathrm{yr}^{-1}$ ) & 3 \\
\hline Number of DHC customers & 500 \\
\hline Average length of distrib. Network per customer (m) & 10 \\
\hline
\end{tabular}

\section{References}

Aboolian, R., Berman, O., Krass, D., 2007. Competitive facility location model with concave demand. European Journal of Operational Research 181 (2), 598-619.

Appa, G.M., Giannikos, I., 1994. Is linear programming necessary for single facility location with maximin of rectilinear distance?. Journal of Operational Research Society 45, 97-107.

Arostegui, M.A., Kadipasaoglu, S.N., Khumawala, B.M., 2006. An empirical comparison of tabu search, simulated annealing, and genetic algorithms for facilities location problems. International Journal of Production Economics 103 (2), 742-754.

Badri, M.A., 1999. Combining the analytic hierarchy process and goal programming for global facility location-allocation problem. International Journal of Production Economics 62 (3), 237-248.

Biezma, M.V., San Cristobal, J.R., 2006. Investment criteria for the selection of cogeneration plants-a state of the art review. Applied Thermal Engineering 26 (5-6), 583-588.

Brimberg, J., Juel, H., 1998. A bicriteria model for locating a semidesirable facility in the plane. European Journal of Operational Research 106 (1), 144-151.

Cooper, L., 1964. Heuristic methods for location-allocation problems. SIAM Review (6), 37-53.

Cundiff, J.S., Dias, N., Sherali, H.D., 1997. A linear programming approach for designing a herbaceous biomass delivery system. Bioresource Technology 59 (1), 47-55.

Dupont, L., 2008. Branch and bound algorithm for a facility location problem with concave site dependent costs. International Journal of Production Economics 112 (1), 245-254.

Eiselt, H.A., 2007. Locating landfills-optimization vs. reality. European Journal of Operational Research 179 (3), 1040-1049.
Freppaz, D., Minciardi, R., Robba, M., Rovatti, M., Sacile, R., Taramasso, A. 2004. Optimizing forest biomass exploitation for energy supply at a regional level. Biomass and Bioenergy 26 (1), 15-25.

Gallis, C.T., 1996. Activity oriented stochastic computer simulation of forest biomass logistics in Greece. Biomass and Bioenergy 10 (5-6), 377-382.

Haupt, R., Haupt, S., 2004. Practical Genetic Algorithms. Wiley, IEEE, New York.

Klose, A., Drexl, A., 2005. Facility location models for distribution system design. European Journal of Operational Research 162 (1), 4-29.

Lahdelma, R., Salminen, P., Hokkanen, J., 2002. Locating a waste treatment facility by using stochastic multicriteria acceptability analysis with ordinal criteria. European Journal of Operational Research 142 (2), 345-356.

Mitchell, C.P., 2000. Development of decision support systems for bioenergy applications. Biomass and Bioenergy 18 (4), 265-278.

Nagel, J., 2000. Determination of an economic energy supply structure based on biomass using a mixed-integer linear optimization model. Ecological Engineering 16, 91-102.

Nanthavanij, S., Asadathorn, N., 1999. Determination of dominant facility locations with minimum noise levels for the cost contour map. International Journal of Production Economics 60, 319-325.

Nilsson, D., 1999. SHAM-a simulation model for designing straw fuel delivery systems. Part 1: model description. Biomass and Bioenergy 16 (1), 25-38.

Nilsson, D., Hansson, P.A., 2001. Influence of various machinery combinations, fuel proportions and storage capacities on costs for co-handling of straw and reed canary grass to district heating plants. Biomass and Bioenergy 20 (4), 247-260.

Owen, S.H., Daskin, M.S., 1998. Strategic facility location: a review. European Journal of Operational Research 111 (3), 423-447.

Papadopoulos, D.P., Katsigiannis, P.A., 2002. Biomass energy surveying and techno-economic assessment of suitable CHP system installations. Biomass and Bioenergy 22 (2), 105-124.

ReVelle, C.S., Eiselt, H.A., Daskin, M.S., 2008. A bibliography for some fundamental problem categories in discrete location science. European Journal of Operational Research 184 (3), 817-848.

Sahin, G., Sural, H., 2007. A review of hierarchical facility location models. Computers and Operations Research 34 (8), 2310-2331.

Sinclair, P., Lofstedt, R., 2001. The influence of trust in a biomass plant application: the case study of Sutton, UK. Biomass and Bioenergy 21 (3), 177-184.

Tatsiopoulos, I.P., Tolis, A.J., 2003. Economic aspects of the cotton-stalk biomass logistics and comparison of supply chain methods. Biomass and Bioenergy 24 (3), 199-214.

Teitz, M.B., Bart, P., 1968. Heuristic methods for estimating the generalized vertex median on a weighted graph. Operations Research 16 (5), 955-961.

Upreti, B.R., 2004. Conflict over biomass energy development in the United Kingdom: some observations and lessons from England and Wales. Energy Policy 32 (6), 785-800.

Upreti, B.R., Van Der Horst, D., 2004. National renewable energy policy and local opposition in the UK: the failed development of a biomass electricity plant. Biomass and Bioenergy 26 (1), 61-69.

Vaithyanathan, S., Burke, L.I., Magent, M.A., 1996. Massively paralle analog tabu search using neural networks applied to simple plant location problems. European Journal of Operational Research 93 (2), $317-330$ 
Voivontas, D., Assimacopoulos, D., Koukios, E.G., 2001. Assessment of biomass potential for power production: a GIS based method. Biomass and Bioenergy 20 (2), 101-112.

Yang, L., Jones, B.F., Yang, S.H., 2007. A fuzzy multi-objective programming for optimization of fire station locations through genetic algorithms. European Journal of Operational Research 181 (2), 903-915.

Yapicioglu, H., Smith, A.E., Dozier, G., 2006. Solving the semi-desirable facility location problem using bi-objective particle swarm. European Journal of Operational Research 177 (2), 733-749.

Zimmerman, J., 1997. Accounting for Decision Making and Control, second ed Irwin-McGraw-Hill, Boston.

Athanasios A. Rentizelas holds a Mechanical Engineering Degree from the National Technical University of Athens (NTUA), an MSc in Operations Management with distinction from UMIST, Manchester, UK and a PhD on Supply Chain Optimization focused on Biomass from NTUA. $\mathrm{He}$ is currently a Research Engineer in the Industrial Management \& Operational Research Sector of NTUA Mechanical Engineering School and a Lecturer of Operations Research at the University of Piraeus. His research interests and expertise lie in the areas of optimization, supply chain management, logistics, renewable energy sources, investment analysis, and quality management. He has been awarded various academic awards for his exceptional performance during his studies, as well as scholarships for postgraduate studies.

Ilias P. Tatsiopoulos is a Professor in Operations Management and Logistics at the Industrial Management and OR Sector of the National Technical University of Athens (NTUA). He has been active for several years as a Professional Production Engineer in both industrial and consulting firms and he served as a Lecturer in Management Information Systems at the Economic University of Athens. He studied Mechanical and Industrial Engineering at NTUA (1978) and followed Post-graduate studies at the TH Aachen (Germany) and the University of Lancaster (UK) under a NATO grant. He holds a PhD (1983) in Operational Research from the University of Lancaster. $\mathrm{He}$ is a member of the Senate of NTUA, Chairman of the Greek Institute for Production \& Operations Management and member of the board of the Hellenic Management Association (HMA). 